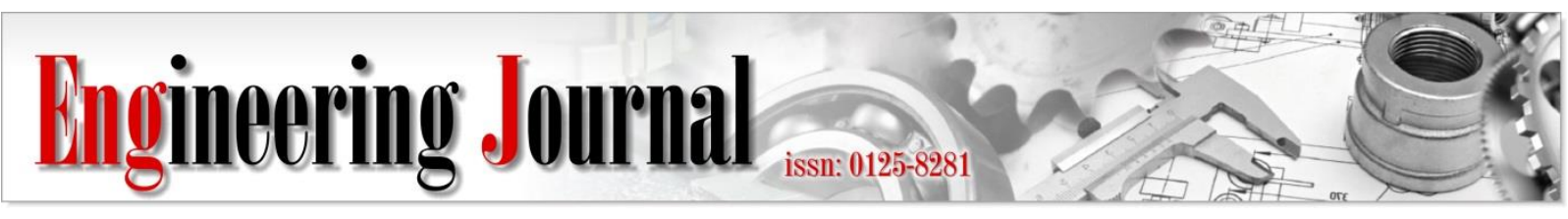

Article

\title{
Optical Fiber Biosensor toward E-coli Bacterial Detection on the Pollutant Water
}

\author{
Hoorieh Fallah ${ }^{1,2, a}$, Tannaz Asadishad ${ }^{2}$, Gholam M. Parsanasab ${ }^{2}$, \\ Sulaiman Wadi Harun ${ }^{3}$, Waleed S. Mohammed ${ }^{4}$, and Moh. Yasin ${ }^{1, b, *}$ \\ 1 Department of Physics, Faculty of Science and Technology, Airlangga University, Surabaya, Indonesia \\ 2 Photonic integrated Circuits laboratory, Electrical Engineering School, Shahid Beheshti University, Iran \\ 3 Photonics Engineering Laboratory, Department of Electrical Engineering, University of Malaya, 50603 \\ Kuala Lumpur, Malaysia \\ 4 Center of Research in Optoelectronics, Communication and Control Systems (BU-CROCCS), School of \\ Engineering, Bangkok University, Pathum Thani, Thailand \\ E-mail: afallah.hoorieh@gmail.com, b, ${ }^{*}$ yasin@fst.unair.ac.id (Corresponding author)
}

\begin{abstract}
In this study, Zinc oxide ( $\mathrm{ZnO})$ nanorods based fiber optic biosensor has been reported for rapid and sensitive detection of Escherichia Coli (E-coli). A thin layer of Gold nanoparticles (Au) (around $50 \mathrm{~nm}$ ) is coated on the tip of a multimode plastic optical fiber. $\mathrm{ZnO}$ Nanorods are grown on Au layer thorough hydrothermal technique. This sensor showed a very fast response within the first 10 second of contacting the present of polluted water with E-coli Different concentrations of E. coli from (1000 to 4000 $\mathrm{CFU} / \mathrm{ml}$ ) have been tested and a sharp trend of sensitivity was observed. This sensing platform shows promising potential for regular water and food quality monitoring of various pathogenic microorganisms.
\end{abstract}

Keywords: Fiber optic biosensor, E. coli bacteria, $\mathrm{ZnO}$ nanorods, Gold(Au) nano layer.

ENGINEERING JOURNAL Volume 25 Issue 12

Received 26 August 2021

Accepted 29 November 2021

Published 30 December 2021

Online at https://engj.org/

DOI:10.4186/ej.2021.25.12.1 


\section{Introduction}

In the recent decades, the high increase in the production of processed food made it hard to sustain low or minimal occurrence of bacterial contamination [1]. Conventional techniques such as plating, polymerase chain reaction (PCR) and enzyme linked immunosorbent assay (ELISA) methods are reliable for the bacterial detection, but they are very time consuming, laborious, expensive and they need costly lab equipment $[2,3]$. Therefore, developing a simple and low-cost technique with rapid response to detect bacterial contamination can be very useful. Several designs have been proposed in literature to reduce the detection cost and improve the device portability. One approach is use a smart phone for fluorescence detection [4]. Though being a practical design, the system still requires high quality film and inserts lenses. Another way to reduce the system cost and complexity is use nanotechnology. This is because nanotechnology could be used to produce accurate, inexpensive, and effective devices for contamination detection such as pathogens and toxins based [5-7]. E. coli, Listeria monocytogenes, Salmonella Typhimurium and Campylobacter are pathogenic strains that cause most of the foodborne disease [8]. Some other type of E. coli is non-pathogenic. That includes E. coli O157: H7, E. coli O153:H2 and E. coli O92: H33. This type of $\mathrm{E}$. coli can cause severe foodborne diseases like diarrhea and acute gastroenteritis [9-10]. Polluted water, unpasteurized milk, meat, and food products are easily affected by the E-coli contamination [11]. The infection can affect the patients with low immunity by causing haemolytic uremic syndrome, thrombotic thrombocytopenic purpura, kidney failure, and even death. Culturing as a common method of E-coli detection is labor-intensive and thus it may delay the patient's treatment and the required disease control [12]. Thus, it is important to establish a rapid, accurate and sensitive detection method for prevention and control of foodborne diseases caused by the bacteria [13].

Integration of optical fiber-based sensor can present a practical solution for biological sensing especially in inaccessible locations. Its mechanical flexibility allows the fiber to transmit optical single over a long distance. These properties allow fiber optics-based sensors to be utilized for the detection of different biological species [14]. Combining nanotechnology and optical fiber for optical sensing has been demonstrated in many literatures. For instance, light scattering from $\mathrm{ZnO}$ nanorods grown on the side of optical fiber has been used for humidity sensing [15]. The presence of nanorods scatters the incident light at angles larger than the critical angle between the surrounding and the cladding [16]. That allows the excitation of cladding modes inside the fiber. Mechanically polished D-shaped fiber was used to enhance the interaction between the evanescent beam and the nanorods grown on the flat surface for ammonia detection application [17]. Utilizing long period gratings, ethanol vapor sensors were fabricated with $\mathrm{ZnO}$ nanorods grown on optical fibers [18]. Mechanical polishing can be avoided by thinning the optical fiber through thermal tapering prior to $\mathrm{ZnO}$ nanorod growth, which allows a larger evanescent tail in the ambient [19].

Recently, utilization of nanomaterial (such as $\mathrm{ZnO}$ nanorods) for fiber optic biosensors has been used to improve the sensitivity and biocompatibility of several sensing platforms [20-21]. $\mathrm{ZnO}$ has unique properties that make it ideal material for electronics and photonics sensing device [22]. This is due to the absence of centre of symmetry in Wurties structure, along with large electromechanical coupling, and wide band gap (3.37 ev [23]. In addition, high surface area to volume ratio causes highly sensitive and efficient light absorption or electron transfer [24]. Different fabrication techniques for $\mathrm{ZnO}$ nanostructures realization were demonstrated including sol-gel technique and hydrothermal method [25]. Hydrothermal method is simple, low cost and has high efficiency [26].

$\mathrm{ZnO}$ rods have also been used in combination with gold or silver films or nanoparticles to create a more efficient biosensors based on surface plasmonic resonance (SPR) and surface-enhanced Raman scattering (SERS) optical methods. For example, to detect Neisseria meningitidis, a think film of $\mathrm{ZnO}$ was deposited on glass prism, coated with Au nanolayers [27]. The developed SPR biosensors showed good sensitivity in the range of target DNA concentration 10-180 ng/ul [27]. ZnO were used as passive component to reduce losses in the optical signal and better attachment of Au nanostructure in SPR biosensor [28]. $\mathrm{ZnO}$ applied on $\mathrm{Au}$ nanoparticles layer increases the active surface area and SPR signals. [29-32]. In label-free detection, the detected signal is generated directly with the on-site interaction of the analyte and the respective transducer. Recombination technique of bioluminescent cells and bioluminescent signal on optical fiber is used to detect an Escherichia coli strain [33].

Here, hydrothermal method is used to grow $\mathrm{ZnO}$ nanorods on a plastic optical fiber tip that is coated with a thin layer of $\mathrm{Au}$ for a potential rapid detection of $\mathrm{E}$. coli bacteria. In literature the use of $\mathrm{ZnO}$ with metal nanoparticle was demonstration for photoelectrochemical bionsensing applications [34]. IN this paper, $\mathrm{ZnO}$ nanorods are used here mainly to scatter cause surface optical back scattering which amplitude is strongly dependent on the surrounding. The gold layer is mainly added to increase the reflected signal back to the detector beyond the noise level in order to enhance coupling to the fiber and to reduce ambient effect. The sensing mechanism for the proposed sensor counts on the change of the backscattered light intensity due to the presence of E. Coli in the solution surrounding the $\mathrm{ZnO}$ nanorods. The increase of the solution effective index as well as the deposition of E. Coli on the rods reduces the amount of scattering and hence increases the signal strength. The orientation of the light source to form large angle minimizes the source effect on the signal. The proposed design is simple, low cost and can be used 
for rapid detection of E. coli contamination in solution. It is worth mentioning that the work did not include surface functionalization with $\mathrm{E}$. coli anti-body due to the lack of the substance at the time of paper preparation. The work however shows a clear possibility for using this technique for future expansion for specificity of detection.

\section{Experimental Arrangement}

\subsection{Material}

A step index Multimode plastic optical fiber (core diameter $125 \mu \mathrm{m}$, FT-430 M-10,10TND, CG20) was used in the experiment. For the hydrothermal growth, Ethanol [C2H6O,99.7\%purity], Zinc acetate dehydrate [ $\mathrm{Zn}(\mathrm{CH} 3) 2,2 \mathrm{H} 2 \mathrm{O}$, Merck, 99. \% Purity], Zinc Nitrate $[\mathrm{Zn}(\mathrm{NO} 3) 2, \quad 6 \mathrm{H} 2 \mathrm{O}, \quad$ Aldrich, $99 \%]$ and Hexamethylenetetramine [CH12N4, Carlo Erba, 99.5\%)] were used. To enhance the reflection signal, a thin layer of Gold (Au) nanoparticle was sputtered to an approximate thickness of $50 \mathrm{~nm}$. The test samples were prepared by a mixture of Escherichia coli (E. coliATCC25922), and deionized water.

\subsection{Synthesis of $\mathrm{ZnO}$ Nanrods}

To grow $\mathrm{ZnO}$ nanorods on a plastic optical fiber (POF), a modified hydrothermal growth method was used as suggested by Ref [33]. The process starts by seeding the substrate with $\mathrm{ZnO}$ nanocrystals followed by a controlled hydrothermal growth process [35]. For the seeding process, $1 \mathrm{mM}$ zinc acetate solution prepared in $20 \mathrm{ml}$ ethanol was used. The cleaned fibers were then placed on a hot plate at $60^{\circ} \mathrm{C}$. A $100 \mu$ l solution of the zinc acetate was then dropped on the fiber for several times. The hydrothermal growth of the nanorods on the tip of the fiber was prepared by a solution containing 10 $\mathrm{mM}$ zinc nitrate hexahydrate and $10 \mathrm{mM}$ hexamethylenetetramine. The sample was then kept in the prepared solution inside an oven at $95^{\circ} \mathrm{C}$. The process time was set to eight hours based on earlier published research work by the authors. In order to maintain a constant growth rate of the nanorods, the solution was replaced every five hours. Finally, the fiber tip with $\mathrm{ZnO}$ nanorods growth was rinsed thoroughly with DI water to wash the residues.

\subsection{Fabrication of Optical Fiber Biosensor}

The probe of fiber optic biosensor was fabricated using a multimode step index coreless plastic fiber (POF). First, the polymer protection layer of the POF was removed. The fiber tip was then polished uniformly. The plastic fiber (without coating layer) was then thoroughly cleaned with deionized water and methanol. It was then dried at the $25^{\circ} \mathrm{C}$. Second, a $50 \mathrm{~nm}$ Gold (Au) layer was sputter coated on the plastic fiber. To do so, a thin chromium (Cr) layer $(\sim 5 \mathrm{~nm})$ was first deposited on the optical fiber surface to provide stronger attachment of $\mathrm{Au}$ in the sputtering process. The coated fibers were then seeded with zinc acetate as in the synthesis process. However, in order to have a uniform surface coating, the process was repeated for seven times at a speed of $9 \mathrm{rpm}$. Finally, $\mathrm{ZnO}$ nanorods were grown using the modified hydrothermal method explained in Section 2.2. Figure 1 shows a schematic diagram of the fabricated fiber based optical sensor. The figure shows the two main mechanism of the sensor where the incident light is refracted from the side at a large angle where it should be generally totally internally reflected at the tip and leak from the other side. The present of $\mathrm{ZnO}$ rods however allows backscattering to be guided and collected by the fiber. The Gold layer pushes the small reflected signal with an DC level in order to reduce the detection noise.

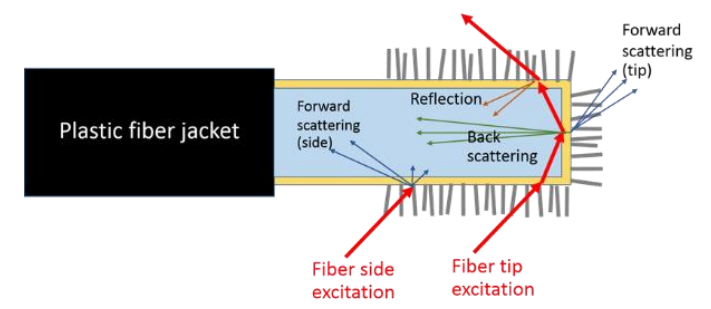

Fig. 1. The fabricated schematic of Optical fiber with $\mathrm{Au}$ layer and Zno Nrs.

\subsection{Bacteria Cell Preparation}

E. coli were grown on a nutrient agar plate at $37^{\circ} \mathrm{C}$ for 24 hours. A loop full seed of microorganisms on nutrient agar plate was inoculated into a $250-\mathrm{ml}$ shaking flask containing $50 \mathrm{ml}$ of nutrient broth medium. The flask was cultivated for eight hours at $37{ }^{\circ} \mathrm{C}$ on a rotary shaker operating at $110 \mathrm{rpm}$. The bacterium cells were then harvested by centrifugation at $12000 \mathrm{~g}$ for 5 minutes at $4{ }^{\circ} \mathrm{C}$ and washed twice with $0.9 \%$ saline solution. The resulting cells were transferred in sterile saline and the optical density $(O D 600 \mathrm{~nm})$ of the suspension was adjusted at 0.13 which is equal to $1.5 \times 108 \mathrm{CFU} / \mathrm{ml}$.

\subsection{Experimental Characterization Setup}

A schematic diagram of the optical characterization setup to test the response of the proposed biosensor is shown in Fig. 2. As shown in the figure, the prepared $\mathrm{POF}$ with $\mathrm{Au}$ layer and $\mathrm{ZnO}$ nanorords is placed inside the glass chamber. The chamber is filled with water polluted by E-coil. Polychromatic unpolarized light from a fiber coupled Tungsten Halogen source (Ocean Optics, HL-2000, wavelengths range, $360-2400 \mathrm{~nm}$, output $6.7 \mathrm{~mW}$ ) is aligned such that the light is incident at the flat POF tip at an angle of $45^{\circ}$ through the transparent glass chamber. The output signal is collected from the other side of the POF. The signal is sent to an optical spectrometer (Avaspec-uls2048*64 TEC-EVO, Wavelength range $200-1160 \mathrm{~nm}$, Resolution, $0.09-20 \mathrm{~nm}$ ). The angle is selected based on earlier optimization for 
back scattering by the $\mathrm{ZnO}$ nanorods [35]. The reflection of the scattered light from $\mathrm{ZnO}$ nanorods at varied concentration of the polluted solution is recorded.

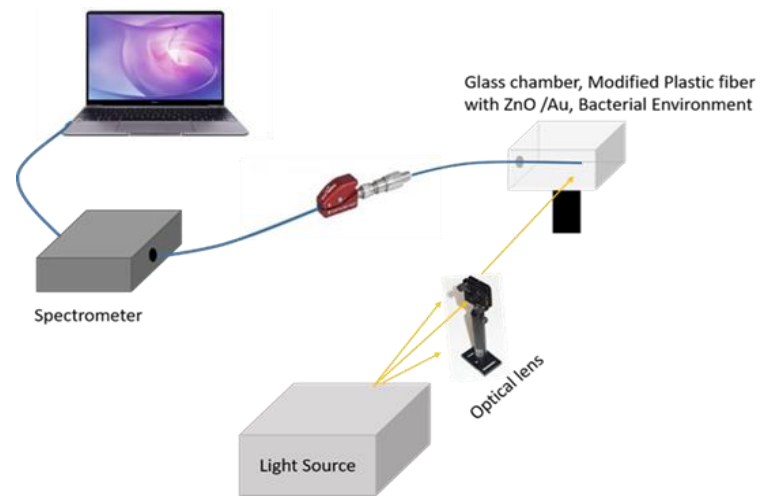

Fig. 2. The proposed optical setup to detect E. coli by the optical fiber sensor.

\section{Results and Discussion}

The thickness of the deposited Au layer on the tip of the plastic fiber is estimated to be approximately $50 \mathrm{~nm}$ [36]. This thickness was selected since it is within the optimum range for obtaining SPR signal after light propagation in the gold film. When the SPR is not utilized, this thickness ensures a sufficient signal strength to reach the nanorods while sustaining enough DC level to avoid the noise limit of the detector. The growth time of $\mathrm{ZnO}$ nanorods on the tip of plastic fiber is set at optimum duration of eight hours, based on the authors' previous work [36]. The growth condition is optimized to maximize the backscattering. SEM images of the grown $\mathrm{ZnO}$ are shown in Figs. 3(a)-(b). The images show a uniform $\mathrm{ZnO}$ nanorods growth and acceptable aligned. A clear SEM image of the tip could not be obtained due to technical difficulty in the SEM session. However, it is clearly shown that the $\mathrm{ZnO}$ growth extends to all sides of the fiber. This can have negative and positive effects on the results. It also allows for side excitation of the signal inside the fiber. The extension of the rods to the side contributes to more leakage and hence lower signal. On the other hand, it does enhance the contrast due to the change in backscattering light caused by the presence of E. coli. Figure 3(c) shows the XRD graph of hexagonal wurtzite morphology of $\mathrm{ZnO}$ nanorods. The maximum XRD peak intensity is found at $34.42^{\circ}$ corresponding to the 002 plane of $\mathrm{ZnO}$. This indicates that the grown $\mathrm{ZnO}$ nanorods is mostly oriented along the [002] direction.
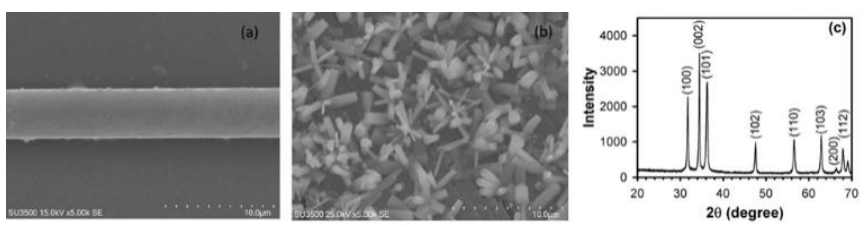

Fig. 3. (a) SEM images of $\mathrm{ZnO}$ nanorods on the $\mathrm{Au}$ surface top view, (b) cross Section, (c) XRD pattern
In the experiment, the incident light refracts (neglecting lensing effect of the fiber side) on the tip at large angles (larger than the critical angle). The presence of the $\mathrm{ZnO}$ nanorods on the tip of the fiber causes two forms of scattering: forward and backward. Forward scattering tends to reduce the reflected signal. The back scattered light reaches the detector (the cone that forms large angles with the side of the fiber). The part of the light that total internally reflected from the tip leaks strongly due to the small angle it forms with the fiber side. Hence, it has minimal effect at the detector. The growth of $\mathrm{ZnO}$ on the sides can as well enhance the leakage of this signal. It is typically desired to have backscattering signal only. Since backscattering signal can be weak, a gold layer was added to slightly increase this background signal beyond the detector noise limits. The total background power caused by this mechanism is referred to as $P_{R}=P_{\text {fr }}-P_{\text {fwd. }}$. Here, $P_{\text {fr }}$ is the power that reaches the detector due to only Fresnel reflection off the fiber tip (without rods). $P_{\text {fwd }}$ is the estimated reduction due to forward scattering of the rods at the fiber tip and sides. Backward scattering, $\mathrm{P}_{\mathrm{bkd}}$, on the other hand is assumed to be guided to the other end of the fiber and reaches the detector due to the large angle it makes with fiber sides. The total recorded power in this case is defined as:

$$
\mathrm{P}_{\text {total }}=\mathrm{P}_{\mathrm{bkd}}+\mathrm{Pfr}-\mathrm{P}_{\mathrm{fwd}}
$$

The main hypothesis behind the selected configuration is to minimize the effect of tip reflection $P_{R}$ due to the strong leakage while propagating to the output tip within a power limit above the noise level. That should hypothetically enhance the contrast of the backscattering signal and, hence, the sensitivity of the device. Practically, the divergence of the incident beam as well as the non-uniformity of the POF tip might cause higher reflected signal than desired. This increases the effect of the forward scattering on the detected signal. In principle, the Fresnel reflection term $\left(\mathrm{P}_{\mathrm{fr}}\right)$ and the forward scattering reduction can be written as:

$$
\mathrm{P}_{\mathrm{fr}}-\mathrm{P}_{\mathrm{fwd}}=\mathrm{P}_{-} 0(\mathrm{R}-\mathrm{Csc}) \exp (-\alpha \mathrm{L})
$$

Here, $\mathrm{R}$ and Csc are the Fresnel reflectance and forward scattering coefficients. The constant $\alpha$ is the leakage coefficient due to reflection at the side of the fiber and $\mathrm{L}$ is the POF length. Hence, theoretically the term in Eq. (2) should be maintained at a minimal effect only to a limit above the noise level.

In this study, to characterize the fiber optic biosensor, the reflection spectra from the light scattered by the nanorods are obtained for different concentrations of E. coli $(1000,2000,3000$, and 4000 $\mathrm{CFU} / \mathrm{ml}$ ). To have more comparative results, $\mathrm{ZnO}$ nanorods were grown in the middle part of plastic fiber using the same growth methodology and characterization technique. Also, to be more accurate in collecting data, an Au layer on optical fiber has been used to detect $\mathrm{E}$ 
Coli. concentration substantially. It is worth mentioning that different concentration of $\mathrm{E}$ Coli bacteria was dissolved in water and the amount of dissolved bacteria in water colony is converted to $\mathrm{CFU} / \mathrm{ml}$ as explained in part 2.4.

The spectra of the different concentrations of E. coli obtained from the tip and middle parts of optical sensor (AU/ZNO/fiber) are shown in Figs. 4(a)-(b). These graphs are calculated as the difference between the measurement with $\mathrm{ZnO}$ nanorods and a control measurement with only $\mathrm{Au}$ coating for each concentration. All the diagrams were recorded after the first 10 second of adding the solution in the flow cell. This time value was obtained from experimental observation for measurements obtained from 1 to 60 seconds. During the observation there were no obvious changes in the recorded spectrum after 10 seconds. It can be observed that the spectrum shows higher intensity as the concentration of E. coli increases. It is shown that the signal collected by the tip excitation is higher compared to side excitation from the middle part. This can be attributed to stronger back scattering from the tip for the eight-hour growth condition. However, side coupling solely depends on forward scattering. The increase of $\mathrm{E}$. Coli concentration mainly increases the effective index of the layer near the $\mathrm{ZnO}$ rods due to the increase probability of $\mathrm{E}$. Coli deposition there. Hence, forward scattering is expected to reduce, and more light is back scattered/reflected.
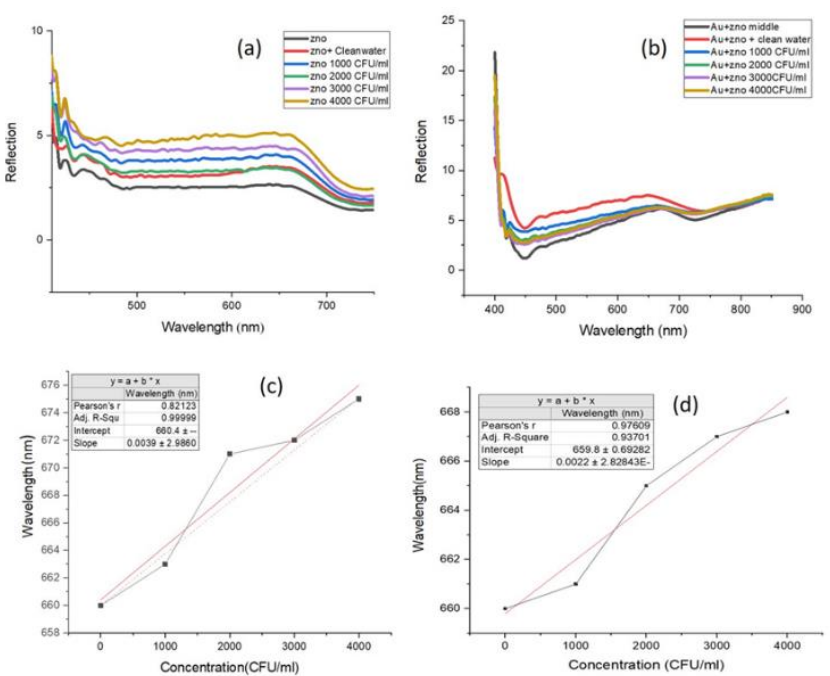

Fig. 4. (a) the reflection scattering spectra from different concentration of E-coli bacteria in the water in contact to the tip of optical fiber ( $\mathrm{Au} / \mathrm{ZnO}$ nanorods/plastic fiber) at visible wavelength (b), the spectra peak for Middle part of optical fiber (Au/ZnO nanorods/plastic fiber). (c) Proportional shift in of $\mathrm{Au} / \mathrm{ZnO} /$ Fiber optic sensor vs. concentrations of E. coli along with standard deviation as error bars. (d) Proportional shift in of $\mathrm{Au} / \mathrm{ZnO} /$ Fiber optic middle part of Fiber.

In Figs. 4(c)-(d), the shift of the anomalies near 650 $\mathrm{nm}$ were investigated versus the effect of the Ecoli concentration. The corresponding shift for different E. coli concentration by the Au/ Zno nanorods optical fiber-based sensor shows larger slope for the fiber tip compared to the side excitation $\left(3.9^{*} 10^{-3}\right.$ compared to $\left.2.2^{*} 10^{-3} \mathrm{~nm} / \mathrm{CFU} / \mathrm{ml}\right)$. This anomaly might not be due to surface plasmon resonance (SPR) between the coated gold layer and the $\mathrm{ZnO} /$ water/E.Coli interface. Using Fabry-Perot analysis of a thin gold film, the calculated SPR excitation around $650 \mathrm{~nm}$ at the designated incident angle theoretically requires a cladding effective index around 1.2. This cannot be the case due the higher refractive indices of both $\mathrm{ZnO}$ and water. Also, it is most likely not due to the increase in the absorbance by the E. Coli as for the used concentration, E. coli absorption in this wavelength range is small and nearly flat [37]. Hence, the anamoly can be mainly due to the change of the contribution of the electrical dipole in Mie scattering by the $\mathrm{ZnO}$ rods with the presence of E. coli. The fact that E. coli has an average length of approximately $2 \mu \mathrm{m}$, could as well enhance the backscattering.

For the intensity modulation, the graphs in Figs. 5(a)-(b) demonstrate the average reflectance measured by averaging the measurement over a wavelength range from $500 \mathrm{~nm}$ to $600 \mathrm{~nm}$. The plots show almost linear increase with the concentration of E. Coli. The slope for the tip excitation is clearly higher than that of side excitation $\left(4.4^{*} 10^{-4}\right.$ compared to $2.5^{*} 10^{-4}$ unit $\left./ \mathrm{CFU} / \mathrm{ml}\right)$. Both wavelength shift and intensity modulation measurements show higher sensitivity for the tip excitation compared to side excitation. The slope in both schemes is around 1.7 times higher for tip excitation.).
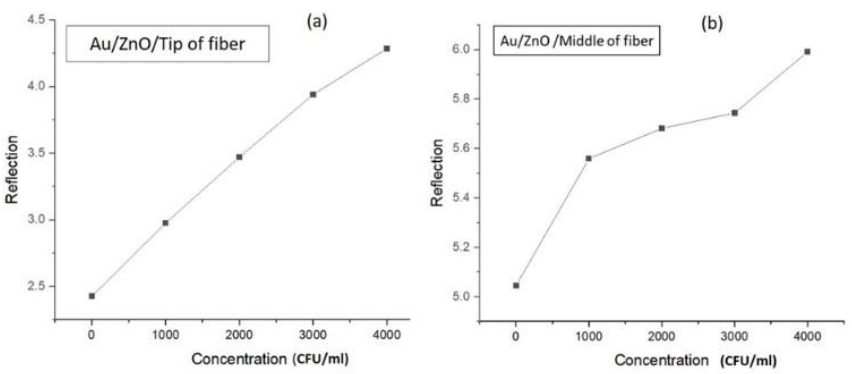

Fig. 5. (a) Different concentrations of E.coli Vs reflection for the tip of fiber, (b) middle part of fiber.

\section{Conclusion}

This work demonstrated the utilization of light scattering by $\mathrm{ZnO}$ nanorods coated on the POF tip for rapid detection of E-coli in water. A thin layer of gold was coated on the fiber to slightly increase the background signal beyond the detector noise limit. Both backward scattering from the fiber tip as well as side forward scattering by side excitation showed almost linear increase of the total reflection with the increase of E. coli concentration. Though, scattering by the fiber tip showed higher sensitivity, 1.7 times, compared to side excitation. This optical biosensor is a simple and low cost system utilizing simply hydrothermal growth of $\mathrm{ZnO}$ nanorods with good uniformity that was confirmed by SEM and XRD analysis. 


\section{Acknowledgement}

We would like to thank Airlangga University for financially supporting this project, and also Photonic Integrated Circuits laboratory at Shahid Beheshti University.

\section{References}

[1] B. D. Gupta and R. Kant, "Recent advances in surface plasmon resonance based fiber optic chemical and biosensors utilizing bulk and nanostructures," Optics \& Laser Technology, vol. 101, pp. 144-161, 2018.

[2] L. He, Q. Pagneux, I. Larroulet, et al. "Label-free femtomolar cancer biomarker detection in human serum using graphene-coated surface plasmon resonance chips," Biosensors and Bioelectronics, vol. 89, pp. 606-611, 2017.

[3] M. Pan, K. Lee, and L. Wang, "Chip-based digital surface plasmon resonance sensing platform for ultrasensitive biomolecular detection," Biosensors and Bioelectronics, vol. 91, no. 580-587, 2017.

[4] M. Maarouf, A. Zeinhomab, Y. Wangac, Y. Songa, M. J. Zhud, Y. Lina, and D. Duae, "A portable smart-phone device for rapid and sensitive detection of E. coli O157:H7 in yoghurt and egg," Biosensors and Bioelectronics, vol. 99, no. 15, pp. 479485, 2018.

[5] M. Couture, S. S. Zhao, and J. F. Masson, "Modern surface plasmon resonance for bioanalytics and biophysics," Phys. Chem. Chem. Phys., vol. 15, pp. 11190-11216, 2013.

[6] Q. Zhou, Y. Lin, K. Zhang, M. Li, and D. Tang, "Reduced graphene oxide/BiFeO3 nanohybridsbased signal-on photoelectrochemical sensing system for prostate-specific antigen detection coupling with magnetic microfluidic device," Biosensors and Bioelectronics, vol. 101, no. 15, pp. 146152, 2018.

[7] Y. Wang and E. C. Alocilja, "Gold nanoparticlelabeled biosensor for rapid and sensitive detection of bacterial pathogens," Journal of Biological Engineering, vol. 9, 2015, Art. no. 16.

[8] S. Kaushik, U. K. Tiwari, S. S. Pal, R. K. Sinha, "Rapid detection of Escherichia coli using fiber optic surface plasmon resonance immunosensor based on biofunctionalized Molybdenum disulfide (MoS2) nanosheets," Biosensors and Bioelectronics, vol. 126, pp. 501-509, 2019.

[9] J. B. Kaper, J. P. Nataro, and H. L. Mobley, "Pathogenic escherichia coli," Nature Reviews Microbiology, vol. 2, no. 2, pp. 123-140, 2004.

[10] H. Pennington, "Escherichia coli O157," The Lancet, vol. 376, no. 9750, pp. 1428-1435, 2010.

[11] K. Wells, "Placement of emotionally disturbed children in residential treatment: A review of placement criteria," American Journal of Orthopsychiatry, vol. 61, no. 3, pp. 339-347, 1991.

[12] C. Zhou, H. Zou, M. Li, C. Sun, D. Ren, and Y. Li, "Fiber optic surface plasmon resonance sensor for detection of E. coli O157: $\mathrm{H} 7$ based on antimicrobial peptides and AgNPs-rGO," Biosensors and Bioelectronics, vol. 117, pp. 347-353, 2018.

[13] B. Balakrishnan, S. Barizuddin, T. Wuliji, and M. El-Dweik, "A rapid and highly specific immuno fluorescence method to detect Escherichia coli O157:H7 in infected meat samples," Int. J. Food Microbiol., vol. 231, pp. 54-62, 2016.

[14] H. Y. Lin, Y. C. Tsao, W. H. Tsai, Y. W. Yang, T. R. Yan, and B. C. Sheu, "Development and application of side-polished fiber immunosensor based on surface plasmon resonance for the detection of Legionella pneumophila with halogens light and 850 nm-LED," Sensors and Actuators A: Physical, vol. 138, no. 2, pp. 299-305, 2007.

[15] Y. Liu, Y. Zhang, H. Lei, J. Song, H. Chen, and B. $\mathrm{Li}$, "Growth of well-arrayed $\mathrm{ZnO}$ nanorods on thinned silica fiber and application for humidity sensing," Opt. Express, vol. 20, pp. 19404-19411, 2012.

[16] H. Fallah, M. Chaudhari, T. Bora, S. W. Harun, W. S. Mohammed, and J. Dutta, "Demonstration of side coupling to cladding modes through zinc oxide nanorods grown on multimode optical fiber," Optics Letters, vol. 38 no. 18, pp. 3620-3622, 2013.

[17] A. O. Dikovska, G. B. Atanasova, N. N. Nedyalkov, P. K. Stefanov, P. A. Atanasov, E. I. Karakoleva, and A. T. Andreev, "Optical sensing of ammonia using $\mathrm{ZnO}$ nanostructure grown on a side-polished optical-fiber," Sens. Actuators B, vol. 146, pp. 331336, 2010.

[18] M. Konstantaki, A. Klini, D. Anglos, and S. Pissadakis, "An ethanol vapor detection probe based on a $\mathrm{ZnO}$ nanorod coated optical fiber long period grating," Opt. Express, vol. 20, pp. 8472-8484, 2012.

[19] M. Batumalay, Z. Harith, H. A. Rafaie, F. Ahmad, M. Khasanah, S. W. Harun, R. M. Nord, and H. Ahmad, "Taperedplasticoptical fiber coated with $\mathrm{ZnO}$ nanostructures for the measurement of uric acid concentrations and changes in relative humidity," Sens. Actuators A, vol. 254, pp. 10871090, 2007.

[20] I. Kant, Kant: The Metaphysics of Morals. Cambridge University Press, 2017.

[21] S. Nayak, C. Mahender, A. Soam, and J. Nanda, "Structural and optical studies of $\mathrm{BiFeO}_{3} @ \mathrm{SiO}_{2}$ core/shell nanoparticles," Materials Research Express, vol. 4, no. 10, p. 105029, 2017.

[22] N. Izu, S. Nishizaki, T. Itoh, M. Nishibori, W. Shin, and I. Matsubara, "Gas response, response time and selectivity of a resistive $\mathrm{CO}$ sensor based on two connected $\mathrm{CeO}_{2}$ thick films with various particle sizes," Sensors and Actuators B: Chemical, vol. 136 no. 2, pp. 364-370, 2009. 
[23] S. Baruah and J. Dutta, "Hydrothermal growth of $\mathrm{ZnO}$ nanostructures," Science and Technology of Advanced Materials, vol. 10 no. 1, p. 013001, 2009.

[24] T. Bora, H. Fallah, M. Chaudhari, T. Apiwattanadej, S. W. Harun, W. S. Mohammed, and J. Dutta, "Controlled side coupling of light to cladding mode of $\mathrm{ZnO}$ nanorod coated optical fibers and its implications for chemical vapor sensing," Sensors and Actuators B: Chemical, vol. 202, pp. 543-550, 2014.

[25] W. Shi, S. Song, and H. Zhang, "Hydrothermal synthetic strategies of inorganic semiconducting nanostructures," Chemical Society Reviews, vol. 42, no. 13, pp. 5714-5743, 2013.

[26] Q. Yang, et al., "Metal oxide and hydroxide nanoarrays: Hydrothermal synthesis and applications as supercapacitors and nanocatalysts," Progress in Natural Science: Materials International, vol. 23, no. 4, pp. 351-36, 2013.

[27] G. Kaur, A. Paliwal, M. Tomar, and V. Gupta, "Detection of Neisseria meningitidis using surface plasmon resonance based DNA biosensor," Biosens. Bioelectron., vol. 78, pp. 106-110, 2016.

[28] A. García-Marín, J. M. Abad, E. Ruiz, E. Lorenzo, J. Piqueras, and J. L. Pau, "Glutathione immunosensing platform based on total internal reflection ellipsometry enhanced by functionalized gold nanoparticles," Anal. Chem., vol. 86, pp. 49694976, 2014.

[29] A. Choi, K. Kim, H.-I. Jung, and S. Y. Lee, " $\mathrm{ZnO}$ nanowire biosensors for detection of biomolecular interactions in enhancement mode," Sens. Actuators B, vol. 148, pp. 577-582, 2010.

[30] P. Sanguino, T. Monteiro, S. R. Bhattacharyya, C. J. Dias, R. Igreja, and R. Franco, " $\mathrm{ZnO}$ nanorods as immobilization layers for interdigitated capacitive immunosensors," Sens. Actuators B: Chem., vol. 204, pp. 211-217, 2014.
[31] Q. Tao, S. Li, Q. Y. Zhang, D. W. Kang, J. S. Yang, W. W. Qiu, and K. Liu, "Controlled growth of $\mathrm{ZnO}$ nanorods on textured silicon wafer and the application for highly effective and recyclable SERS substrate by decorating Ag nanoparticles," Mater. Res. Bull., vol. 54, pp. 6-12, 2014.

[32] R. Niepelt, U. C. Schröder, J. Sommerfeld, I. Slowik, B. Rudolph, R. Möller, B. Seise, A. Csaki, W. Fritzsche, and C. Ronning, "Biofunctionalization of zinc oxide nanowires for DNA sensory applications," Nanoscale Res. Lett., vol. 6, 2011, Art. no. 511.

[33] I. Biran, D. M. Rissin, E. Z. Ron, and D. R. Walt, "Optical imaging fiber-based live bacterial cell array biosensor," Anal. Biochem., vol. 315, pp. 106-113, 2003.

[34] Y. Li, W. Wang, H. Gong, J. Xu, Z. Yu, Q. Wei and D. Tang "Graphene-coated copper-doped $\mathrm{ZnO}$ quantum dots for sensitive photoelectrochemical bioanalysis of thrombin triggered by DNA nanoflowers," J. Mater. Chem. B, vol. 9, pp. 6818-6824, 2021.

[35] H. Fallah, M. Afra, E. Mohajerani, and W. S. Mohammad, "Utilization of $\mathrm{ZnO}$ nanorods growth on a tip of plastic optical fiber toward the realization of low-cost $\mathrm{CO}$ and $\mathrm{CO}_{2}$ gas sensor," Journal of Nanophotonics, vol. 11 no. 3, p. 036023, 2017.

[36] H. Suzuki, M. Sugimoto, Y. Matsui, J. Kondoh, "Effects of gold film thickness on spectrum profile and sensitivity of a multimode-optical-fiber SPR sensor," Sensors and Actuators B: Chemical, vol. 132 no. 1, pp. 26-33, 2008.

[37] J. Kiefer, N. Ebel, E. Schlücker, and A. Leipertz, "Characterization of Escherichia coli suspensions using UV/Vis/NIR absorption spectroscopy," Anal. Methods, vol. 2, no. 2, pp. 123-128, 2010.

Dr. Hoorieh Fallah received her M.Sc. degree in Nanotechnology from Asian Institute of Technology, Bangkok, Thailand, 2012. She received her Ph.D. in photonics from University of Malaya, Malaysia. Currently she is working as a researcher at Shahid Behehsti University. Her research focuses on fabrication of Nano fiber optic sensors towards gas detection and biosensing applications.-looking.

Tannaz Asadishad received her M.Sc. degree in Photonics from the laser and plasma research institute of Shahid Behehsti University of Tehran, Iran. She received her BSc. in Physic from the shahid beheshti University. Iran. Her research focuses on biosensors and gas detection.

Dr. Gholam Mohammad Parsanasab received his Ph.D. in Electrical Engineering from Shiraz University, Iran, 2015. He is the assistance Professor of Electrical Engineering at Shahid Beheshti University, Iran, from 2017. His research focuses on integrated photonics devices and materials. Micro and nano cavity lasers and organic photonics. 


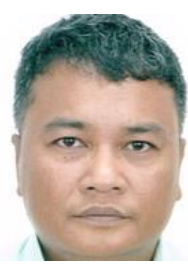

Sulaiman Wadi Harun received his bachelor's degree in Electrical and Electronics System Engineering from Nagaoka University of Technology, Japan, in 1996, and his master's and doctoral degrees in Photonics Technology from the University of Malaya in 2001 and 2004, respectively. He has nearly 20 years of research experience in the development of optical fiber devices including fiber amplifiers, fiber lasers, and fiber optic sensors. Professor Harun has published more than 900 articles in reputable ISI journals, and his papers have been cited more than 8000 times with an hindex of 40, showing the impact on the community. He is the Fellow of the Malaysian Academic of Science, and the founder and honorary advisor for the Optical Society of Malaysia..

Dr. Waleed S. Mohammed graduated from Cairo University in 1996. In 1999 he joined the College of optics and photonics/CREOL, University of Central Florida, USA. He completed his Ph.D. work in optics in 2004. He then joined University of Toronto, as a postdoctoral fellow. In 2007 Dr. Mohammed joined the International school of engineering, Chulalongkorn University, Thailand as an instructor. As of 2010, He joined the school of Engineering, Bangkok University as a research scholar.

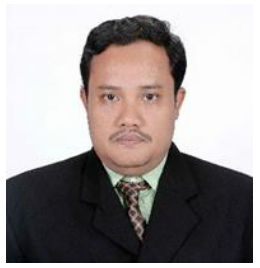

Moh. Yasin received his B.Sc. in 1990 from Airlangga University. He went on to receive M.Sc. and Ph.D. from Gadjah Mada University in 1999 and 2010. His research interests are mainly on fiber optic sensor devices. Currently, he is a full professor at the Faculty of Science and Technology, Airlangga Univesity, Indonesia. He has published more than 200 paper in ISI/Scopus Journal. 